\title{
CR Structures and Twisting Vacuum Spacetimes with Two Killing Vectors and Cosmological Constant: Type II and More Special
}

\author{
Xuefeng Zhang and Daniel Finley \\ Department of Physics and Astronomy, University of New Mexico, Albuquerque, NM \\ 87131 USA \\ E-mail: zxf@unm.edu and finley@phys.unm.edu
}

\begin{abstract}
Based on the CR formalism of algebraically special spacetimes by Hill, Lewandowski and Nurowski, we derive a nonlinear system of two real ODEs, of which the general solution determines a twisting type II (or more special) vacuum spacetime with two Killing vectors (commuting or not) and at most seven real parameters in addition to the cosmological constant $\Lambda$. To demonstrate a broad range of interesting spacetimes that these ODEs can capture, special solutions of various Petrov types are presented and described as they appear in this approach. They include Kerr-NUT, Kerr and Debney/Demiański's type II, Lun's type II and III (subclasses of Held-Robinson), MacCallum and Siklos' type III $(\Lambda<0)$, and the type $N$ solutions $(\Lambda \neq 0)$ we found in an earlier paper, along with a new class of type II solutions as a nontrivial limit of Kerr and Debney's type II solutions. Also, we discuss a situation in which the two ODEs can be reduced to one. However, constructing the general solution still remains an open problem.
\end{abstract}

PACS numbers: 04.20.Jb, 02.40.Tt, 02.30.Hq

Submitted to: Class. Quantum Grav. 


\section{Introduction}

All algebraically special Einstein spaces-vacuum but possibly with a nonzero cosmological constant $\Lambda$ - possess a repeated principal null direction, which generates a foliation of the spacetime by a 3-parameter congruence of shearfree and null geodesics [1, 2]. This 3-dimensional parameter space can be identified with a 3-dimensional real manifold described by the theory of $\mathrm{CR}$ structures with one complex and one real coordinate. CR structures were first introduced by Poincaré and extensively studied by E. Cartan [3, 4]. Good sources of background on the relationship between spacetimes and CR structures can be found, for instance, in the thesis of Nurowski [5]. Recently, Hill, Lewandowski and Nurowski [6] generalized earlier work of [7, 5] to provide a new formulation of twisting algebraically special spacetimes with cosmological constant. It allows a classification of algebraically special spacetimes according to Cartan's classification of 3-dimensional CR structures.

There is indeed a method to determine the equivalence of two solutions of Einstein's equations without having to construct explicit coordinate transformations that map one into the other, the idea of which was originated in work of Cartan and pushed forward by Brans [8], Karlhede [9], and Skea [10, 11]. Cartan also created a method for determining equivalence of two CR manifolds, which is much simpler than the method mentioned above for 4-dimensional manifolds. Because of the correspondence between the two, it allows a considerably simpler approach to determine the equivalence of two twisting algebraically special spacetimes, or, more usefully in this paper, the lack of such an equivalence, thereby guaranteeing that two solutions are distinct. In addition to this important reason for using CR structures, it also provides a different formulation of Einstein's equations, which exhibits certain invariant features that are desirable for calculations, as compared to other formulations. It is favorable to have this invariant approach to study the decomposition of Einstein's equations into some manageable form, and to have one that prefers non-zero values of the twist, since only a very limited number of such spacetimes with non-zero twists, in Petrov types II, III, and N, are actually available for study.

In the theory of exact solutions, Einstein's equations are usually solved under assumptions of the existence of some symmetry group [12, i.e., Killing vectors. For instance, Kerr and Debney [13] have determined all diverging (twisting or not) algebraically special vacuums $(\Lambda=0)$ with three or more Killing vectors. The case with two Killing vectors, however, is still not solved completely. We intend to address this problem in this paper with the extension to include a nonzero cosmological constant.

Building on the work of Hill, Lewandowski, and Nurowski [6], we first present, in

Section 2, the twisting type II vacuum metric formulated according to CR geometry, together with our calculated Weyl scalars. Then in Section 3, we establish the transformation from the CR formalism to the canonical frame that is widely used, e.g., in [12. In Section 4, we generalize the ansatz that was found for twisting type N solutions in [14], thereby reducing the field equations to only two coupled real ODEs for 
two unknown functions of a single variable. From Section 5 to 8, we show, by using the transformation in Section 3, that a large variety of previously-known, twisting solutions of types II, III, as well as D ([12], Chapters 29 and 38), with at least two Killing vectors, correspond to special solutions of these ODEs. Moreover, we study a special case when the number of ODEs can be reduced to one, which generalizes our previous results on type N. Though the general solution is yet to be found, we believe that this extension of ODEs is quite worthwhile and should provide a start for future steps forward in the study of twisting exact solutions.

\section{CR structures and the field equations}

A CR structure 1 is a 3 -dimensional real manifold $M$ equipped with an equivalence class of pairs of 1 -forms $\lambda$ (real) and $\mu$ (complex) satisfying

$$
\lambda \wedge \mu \wedge \bar{\mu} \neq 0
$$

Another pair $\left(\lambda^{\prime}, \mu^{\prime}\right)$ is equivalent to $(\lambda, \mu)$, iff there exist functions $f \neq 0$ (real) and $h \neq 0, g$ (complex) on $M$ such that

$$
\lambda^{\prime}=f \lambda, \quad \mu^{\prime}=h \mu+g \lambda, \quad \bar{\mu}^{\prime}=\bar{h} \bar{\mu}+\bar{g} \lambda .
$$

For our purpose, we further assume [6, 3]

$$
\begin{aligned}
& \mu=\mathrm{d} \zeta, \quad \bar{\mu}=\mathrm{d} \bar{\zeta}, \\
& \mathrm{d} \lambda=\mathrm{i} \mu \wedge \bar{\mu}+(c \mu+\bar{c} \bar{\mu}) \wedge \lambda,
\end{aligned}
$$

where $\zeta$ and $c$ are some complex-valued functions on $M$. Taking the closure of (2), we obtain a reality condition on the derivatives of $c$ :

$$
\partial \bar{c}=\bar{\partial} c
$$

The same function $c$ also appears in the commutation relations of the dual basis of vector fields:

$$
\begin{aligned}
& \left(\partial_{0}, \partial, \bar{\partial}\right) \quad \text { dual to } \quad(\lambda, \mu, \bar{\mu}), \\
& {[\partial, \bar{\partial}]=-\mathrm{i} \partial_{0}, \quad\left[\partial_{0}, \partial\right]=c \partial_{0}, \quad\left[\partial_{0}, \bar{\partial}\right]=\bar{c} \partial_{0} .}
\end{aligned}
$$

There is then the following theorem telling us how to construct an algebraically special spacetime on the basis of $M$.

Theorem 1. [6, 15] The CR structure (1,4) on $M$ can be lifted to a spacetime $\mathcal{M}=M \times \mathbb{R}$ equipped with the metric

$$
\begin{aligned}
& \mathbf{g}=2\left(\theta^{1} \theta^{2}+\theta^{3} \theta^{4}\right), \quad \theta^{1}=\mathcal{P} \mu=\bar{\theta}^{2}, \\
& \theta^{3}=\mathcal{P} \lambda, \quad \theta^{4}=\mathcal{P}(\mathrm{d} r+\mathcal{W} \mu+\mathcal{W} \bar{\mu}+\mathcal{H} \lambda),
\end{aligned}
$$

where $\mathcal{P} \neq 0, \mathcal{H}$ (real) and $\mathcal{W}$ (complex) are arbitrary functions on $\mathcal{M}$. The spacetime (5) admits a geodesic, shearfree and twisting null congruence along the vector field $\partial_{r}$ $(r \in \mathbb{R})$, of which the 3-parameter leaf spaces $(r=$ const.) have the same CR structure

\footnotetext{
${ }^{1}$ All our considerations are local.
} 
CR Structures and Twisting Vacuum Spacetimes: Type II and More Special

as $M$. It further satisfies the Einstein equation $\operatorname{Ric}(\mathbf{g})=\Lambda \mathbf{g}$, iff the metric components can be written as

$$
\begin{aligned}
& \mathcal{P}=\frac{p}{\cos \left(\frac{r}{2}\right)}, \quad \mathcal{W}=\mathrm{i} a\left(\mathrm{e}^{-\mathrm{i} r}+1\right), \\
& \mathcal{H}=\frac{n}{p^{4}} \mathrm{e}^{2 \mathrm{i} r}+\frac{\bar{n}}{p^{4}} \mathrm{e}^{-2 \mathrm{i} r}+q \mathrm{e}^{\mathrm{i} r}+\bar{q} \mathrm{e}^{-\mathrm{i} r}+h, \\
& a=c+2 \partial \log p, \\
& q=\frac{3 n+\bar{n}}{p^{4}}+\frac{2}{3} \Lambda p^{2}+\frac{2 \partial p \bar{\partial} p-p(\partial \bar{\partial} p+\bar{\partial} \partial p)}{2 p^{2}}-\frac{\mathrm{i}}{2} \partial_{0} \log p-\bar{\partial} c, \\
& h=3 \frac{n+\bar{n}}{p^{4}}+2 \Lambda p^{2}+\frac{2 \partial p \bar{\partial} p-p(\partial \bar{\partial} p+\bar{\partial} \partial p)}{p^{2}}-2 \bar{\partial} c,
\end{aligned}
$$

where $c, n$ (complex) and $p$ (real), all functions on $M$ (independent of $r$ ), satisfy the following set of equations:

$$
\begin{aligned}
& \partial \bar{c}=\bar{\partial} c, \\
& {\left[\partial \bar{\partial}+\bar{\partial} \partial+\bar{c} \partial+c \bar{\partial}+\frac{1}{2} c \bar{c}+\frac{3}{4}(\partial \bar{c}+\bar{\partial} c)\right] p=\frac{n+\bar{n}}{p^{3}}+\frac{2}{3} \Lambda p^{3},} \\
& \partial n+3 c n=0, \\
& R_{33}=0 .
\end{aligned}
$$

Here the Ricci tensor component $R_{33}$ as well as the Weyl scalars $\Psi_{2}, \Psi_{3}$ and $\Psi_{4}$ are given by

$$
\begin{aligned}
R_{33}= & \left\{\frac{8}{p^{4}}(\partial+2 c)\left[p^{2}\left(\partial \bar{I}-2 \Lambda(2 \bar{\partial} \log p+\bar{c}) p^{2}\right)\right]\right. \\
+ & \frac{16}{p} \Lambda\left[\left(\partial \bar{\partial}+\bar{\partial} \partial+\bar{c} \partial+c \bar{\partial}+\frac{1}{2} c \bar{c}+\frac{3}{4}(\partial \bar{c}+\bar{\partial} c)\right) p-\frac{n+\bar{n}}{p^{3}}-\frac{2}{3} \Lambda p^{3}\right] \\
+ & \left.\frac{16 \mathrm{i}}{p^{3}} \partial_{0}\left(\frac{n}{p^{3}}\right)\right\} \cos ^{4}\left(\frac{r}{2}\right), \\
\Psi_{2}= & \frac{n}{2 p^{6}}\left(\mathrm{e}^{\mathrm{i} r}+1\right)^{3}, \\
\Psi_{3}= & \left\{\frac{2 \mathrm{i}}{p^{2}}\left[\partial \bar{I}-2 \Lambda(2 \bar{\partial} \log p+\bar{c}) p^{2}\right]\right. \\
& \left.+6 \mathrm{i}(2 \bar{\partial} \log p+\bar{c}) \frac{n}{p^{6}}\left(\mathrm{e}^{2 \mathrm{i} r}-1\right)-4 \mathrm{i} \bar{\partial}\left(\frac{n}{p^{6}}\right)\left(\mathrm{e}^{\mathrm{i} r}+1\right)\right\} \mathrm{e}^{\mathrm{i} r / 2} \cos ^{3}\left(\frac{r}{2}\right),
\end{aligned}
$$




$$
\begin{aligned}
& \Psi_{4}=2 \mathrm{e}^{-\mathrm{i} r / 2} \cos ^{3}\left(\frac{r}{2}\right)\left\{-(2 \bar{\partial} \log p+\bar{c})\left[\partial \bar{I}-2 \Lambda(2 \bar{\partial} \log p+\bar{c}) p^{2}\right] \frac{\mathrm{e}^{2 \mathrm{i} r}-1}{p^{2}}\right. \\
& +(\bar{\partial}+2 \bar{c})\left[\partial \bar{I}-2 \Lambda(2 \bar{\partial} \log p+\bar{c}) p^{2}\right] \frac{\mathrm{e}^{\mathrm{i} r}+1}{p^{2}}+\frac{\mathrm{i}}{p^{2}} \partial_{0} \bar{I} \\
& +\frac{2}{3} \Lambda\left[(\bar{\partial}+\bar{c})(2 \bar{\partial} \log p+\bar{c})+2(2 \bar{\partial} \log p+\bar{c})^{2}\right]-3(2 \bar{\partial} \log p+\bar{c})^{2} \frac{n}{p^{6}} \mathrm{e}^{4 i r} \\
& \left.+\left[(\bar{\partial}-2 \bar{\partial} \log p)(2 \bar{\partial} \log p+\bar{c}) \frac{n}{p^{6}}+3(2 \bar{\partial} \log p+\bar{c}) \bar{\partial}\left(\frac{n}{p^{6}}\right)\right] \mathrm{e}^{3 \mathrm{i} r} \bar{c}^{2}\right] \mathrm{e}^{2 \mathrm{i} r} \\
& +\left[3\left(2 \bar{\partial}^{2} \log p-16(\bar{\partial} \log p)^{2}+2 \bar{c} \bar{\partial} \log p+\bar{c}^{2}\right) \frac{n}{p^{6}}+\frac{(16 \bar{\partial} \log p+\bar{c}) \bar{\partial} n-\bar{\partial}^{2} n}{p^{6}}\right] \mathrm{e}^{\mathrm{i} r} \\
& +\left[3\left(2 \bar{\partial}^{2} \log p-8(\bar{\partial} \log p)^{2}+8 \bar{c} \bar{\partial} \log p-\bar{\partial} \bar{c}-\bar{c}^{2}\right) \frac{n}{p^{6}}+\frac{7(2 \bar{\partial} \log p-\bar{c}) \bar{\partial} n-2 \bar{\partial}^{2} n}{p^{6}}\right\}, \\
& \left.+2\left(\bar{\partial}^{2} \log p-2(\bar{\partial} \log p)^{2}+5 \bar{c} \bar{\partial} \log p-\bar{\partial} \bar{c}-3 \bar{c}^{2}\right) \frac{n}{p^{6}}+\frac{(4 \bar{\partial} \log p-5 \bar{c}) \bar{\partial} n-\bar{\partial}^{2} n}{p^{6}}\right\}
\end{aligned}
$$

with the function $I$ defined by

$$
I=\partial(\partial \log p+c)+(\partial \log p+c)^{2} .
$$

Following the same procedure as [6], which uses Cartan's structure equations to calculate the curvature tensor, we present our calculated $R_{33}, \Psi_{3}$ and $\Psi_{4}$ above with nonzero $\Lambda$ and $n$, as a complement to [6]. Moreover, to facilitate future calculations, we have arranged the expression of $R_{33}$ so that its second square bracket can be immediately removed by the field equation (12), whereas the terms $\partial \bar{I}-2 \Lambda(2 \bar{\partial} \log p+\bar{c}) p^{2}$ are made prominent as they also appear in $\Psi_{3}$ and $\Psi_{4}$.

To solve the field equations (11,14) in practice, one needs to introduce a real coordinate system $(x, y, u)$ on $M$ such that

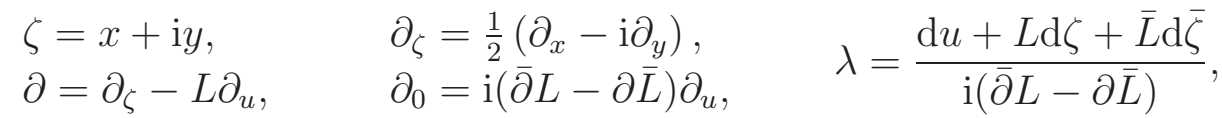

with $L=L(\zeta, \bar{\zeta}, u)$ a complex-valued function [16] satisfying

$$
\bar{\partial} L-\partial \bar{L} \neq 0
$$

which is needed for a nonzero twist (cf. (27)). In addition, $L$ relates to the function $c$ by

$$
c=-\partial \ln (\bar{\partial} L-\partial \bar{L})-\partial_{u} L,
$$

as imposed by the commutation relations (44). Hence generally, the system (11) 14) are in fact PDEs for the unknown functions $L, n$ and $p$ of the coordinate variables $(\zeta, \bar{\zeta}, u)$.

For other possible coordinate choices, the metric (1020) admits the following coordinate freedom ([17], see Section 2.6):

$$
r^{\prime}=r, \quad \zeta^{\prime}=f(\zeta), \quad u^{\prime}=F(\zeta, \bar{\zeta}, u), \quad \partial_{u} F \neq 0,
$$


with $f(\zeta)$ holomorphic and $F$ a real-valued function, which generates the transformation laws

$$
\begin{aligned}
& \mu^{\prime}=f^{\prime} \mu, \quad \lambda^{\prime}=f^{\prime} \bar{f}^{\prime} \lambda, \quad f^{\prime} \equiv \mathrm{d} f / \mathrm{d} \zeta, \\
& \partial^{\prime}=\frac{1}{f^{\prime}} \partial, \quad \partial_{0}^{\prime}=\frac{1}{f^{\prime} \bar{f}^{\prime}} \partial_{0}, \quad\left[\partial^{\prime}, \bar{\partial}^{\prime}\right]=-\mathrm{i} \partial_{0}^{\prime}, \quad\left[\partial_{0}^{\prime}, \partial^{\prime}\right]=c^{\prime} \partial_{0}^{\prime}, \\
& c^{\prime}=\frac{1}{f^{\prime}} c+\frac{f^{\prime \prime}}{\left(f^{\prime}\right)^{2}}, \quad p^{\prime}=\frac{1}{\left|f^{\prime}\right|} p, \quad n^{\prime}=\frac{1}{\left(f^{\prime} \bar{f}^{\prime}\right)^{3}} n, \\
& L^{\prime}=-\frac{1}{f^{\prime}}\left(\partial_{\zeta} F-L \partial_{u} F\right)=-\frac{1}{f^{\prime}} \partial F, \quad \bar{\partial}^{\prime} L^{\prime}-\partial^{\prime} \bar{L}^{\prime}=\frac{\partial_{u} F}{f^{\prime} \bar{f}^{\prime}}(\bar{\partial} L-\partial \bar{L}) .
\end{aligned}
$$

Note that the function $F$ does not appear above on the level of $c, p$ and $n$, as well as $\partial$ and $\partial_{0}$, the fact of which indicates an invariant feature of the CR formalism. As expected, the field equations for the new $p^{\prime}, c^{\prime}$ and $n^{\prime}$ take on the same form of (11) 14) with $\left(\partial_{0}, \partial, \bar{\partial}\right)$ simply replaced by $\left(\partial_{0}^{\prime}, \partial^{\prime}, \bar{\partial}^{\prime}\right)$. These transformation properties will be used to simplify our metrics.

\section{Transformations to the canonical frame}

Given the algebraically special twisting metric form (1,20) formulated according to CR geometry, it is important to know how it is related to other pre-existing formalisms that have been extensively studied in the past. Here we quote from [12] (p. 439-441) a most commonly used one by Kerr, Debney et al. [18, 19, 20]. For simplicity, we only consider $\Lambda=0$ and follow closely the notation of [12] with sub- or superscript $s$ added to avoid confusion.

Theorem 2. A spacetime admits a geodesic, shearfree and twisting null congruence along the vector field $\partial_{r_{s}}$ and satisfies the Einstein equation $\operatorname{Ric}(\mathbf{g})=0$, iff the metric can be written as

$$
\begin{array}{ll}
\mathbf{g}=2\left(\omega^{1} \omega^{2}-\omega^{3} \omega^{4}\right), & \omega^{1}=-\frac{\mathrm{d} \zeta}{P_{s} \bar{\rho}_{s}}=\bar{\omega}^{2}, \\
\omega^{3}=\mathrm{d} u+L \mathrm{~d} \zeta+\bar{L} \mathrm{~d} \bar{\zeta}, & \omega^{4}=\mathrm{d} r_{s}+W_{s} \mathrm{~d} \zeta+\bar{W}_{s} \mathrm{~d} \bar{\zeta}+H_{s} \omega^{3},
\end{array}
$$

with metric components

$$
\begin{aligned}
& \rho_{s}^{-1}=-\left(r_{s}+\mathrm{i} \Sigma_{s}\right), \quad \frac{2 \mathrm{i} \Sigma_{s}}{P_{s}^{2}}=\bar{\partial} L-\partial \bar{L} \neq 0, \\
& W_{s}=\rho_{s}^{-1} \partial_{u} L+\mathrm{i} \partial \Sigma_{s}, \quad \partial=\partial_{\zeta}-L \partial_{u}, \\
& H_{s}=\frac{1}{2} K_{s}-r_{s} \partial_{u} \log P_{s}-\frac{m_{s} r_{s}+M_{s} \Sigma}{r_{s}^{2}+\Sigma^{2}}, \\
& K_{s}=2 P_{s}^{2} \operatorname{Re}\left[\partial\left(\bar{\partial} \log P_{s}-\partial_{u} \bar{L}\right)\right]
\end{aligned}
$$

such that the functions $m_{s}, M_{s}, P_{s}$ (real) and $L$ (complex), all only dependent on the coordinates $(\zeta, \bar{\zeta}, u)$, satisfy a system of PDEs:

$$
\begin{aligned}
& P_{s}^{-3} M_{s}=\operatorname{Im} \partial \partial \bar{\partial} \bar{\partial} V_{s}, \quad P_{s}=\partial_{u} V_{s}, \\
& \partial\left(m_{s}+\mathrm{i} M_{s}\right)=3\left(m_{s}+\mathrm{i} M_{s}\right) \partial_{u} L, \\
& \partial_{u}\left[P_{s}^{-3}\left(m_{s}+\mathrm{i} M_{s}\right)\right]=P_{s}\left[\partial+2\left(\partial \log P_{s}-\partial_{u} L\right)\right] \partial I_{s},
\end{aligned}
$$


where the function $I_{s}$ is defined by

$$
I_{s}=\bar{\partial}\left(\bar{\partial} \log P_{s}-\partial_{u} \bar{L}\right)+\left(\bar{\partial} \log P_{s}-\partial_{u} \bar{L}\right)^{2}=P_{s}^{-1} \partial_{u} \bar{\partial} \bar{\partial} V_{s} .
$$

Additionally, the Weyl scalar $\Psi_{2}^{s}$ is given by

$$
\Psi_{2}^{s}=\left(m_{s}+\mathrm{i} M_{s}\right) \rho_{s}^{3} .
$$

In this metric form, the coordinates $(\zeta, \bar{\zeta}, u)$ and the function $L$ have been chosen identically with those introduced in (18); hence, each is not given a sub- or superscript $s$. Taking $\Lambda=0$ and by a tedious but straightforward calculation, one can show that the metrics (26,31) and (1120) are equivalent to each other by the transformation [17]

$$
\begin{aligned}
& P_{s}=\frac{2 p}{\mathrm{i}(\bar{\partial} L-\partial \bar{L})}, \\
& r_{s}=-\frac{2 p^{2}}{\mathrm{i}(\bar{\partial} L-\partial \bar{L})} \tan \left(\frac{r}{2}\right), \quad|r|<\pi, \\
& m_{s}=\frac{16(n-\bar{n})}{(\bar{\partial} L-\partial \bar{L})^{3}}, \quad M_{s}=\frac{16(n+\bar{n})}{\mathrm{i}(\bar{\partial} L-\partial \bar{L})^{3}},
\end{aligned}
$$

with the inverse

$$
\begin{aligned}
p & =\frac{\mathrm{i}}{2}(\bar{\partial} L-\partial \bar{L}) P_{s}, \\
r & =2 \arctan \left(-\frac{2}{\mathrm{i}(\bar{\partial} L-\partial \bar{L}) P_{s}^{2}} r_{s}\right), \\
n & =\frac{1}{32}\left(m_{s}+\mathrm{i} M_{s}\right)(\bar{\partial} L-\partial \bar{L})^{3} .
\end{aligned}
$$

In particular, the field equation (28) can be transformed into (12) with $\Lambda=0$, despite their drastically different appearances. Also, one gets $I_{s}=\bar{I}$ when substituting (32) into the definition (31). For more details about these transformations, one may see Section 2.6 of [17]. The relation (35) and (37) together with (20) will be used later to translate known solutions of the canonical field equations (28)

\section{Reductions to ODEs}

Now we go back to the metric (1,20). Following the same idea as [14] for solving the field equations, we assume that the unknowns $p, c$ and $n$ have no $u$-dependence, i.e., $\partial_{0} p=\partial_{0} c=\partial_{0} n=0$. This assumption avoids the involvement of the function $L$ inside the operator $\partial$ since now we have, e.g., $\partial p=\partial_{\zeta} p$. Therefore the system (11]14) becomes effectively PDEs for the unknowns $c, p$ and $n$ instead of $L, p$ and $n$. Once $c=c(\zeta, \bar{\zeta})$ is solved, one may further determine a function $L$ without $u$-dependence from (20) (cf. (49)). Altogether, this means that the resulting spacetime shall possess a Killing vector in the $u$-direction, which is, in fact, an assumption widely used in many research articles on algebraically special solutions (see, e.g., [12] Chapter 29).

We apply the assumption and rewrite the system (11,14) (likewise for $\Psi_{3}$ and $\Psi_{4}$ ) as

$$
\partial_{\zeta} \bar{c}=\partial_{\bar{\zeta}} c,
$$




$$
\begin{aligned}
& 2 \partial_{\zeta} \partial_{\bar{\zeta}} p+\bar{c} \partial_{\zeta} p+c \partial_{\bar{\zeta}} p+\frac{1}{2} c \bar{c} p+\frac{3}{2}\left(\partial_{\zeta} \bar{c}\right) p=\frac{n+\bar{n}}{p^{3}}+\frac{2}{3} \Lambda p^{3}, \\
& \partial_{\zeta} n+3 c n=0 \\
& \left(\partial_{\zeta}+2 c\right)\left[p^{2} \partial_{\zeta} \bar{I}-2 \Lambda\left(2 \partial_{\bar{\zeta}} \log p+\bar{c}\right) p^{4}\right]=0
\end{aligned}
$$

where in the last equation $\left(R_{33}=0\right)$ we have used (12) to simplify the expression of $R_{33}$, and the function $I$ is given by

$$
I=\partial_{\zeta}\left(\partial_{\zeta} \log p+c\right)+\left(\partial_{\zeta} \log p+c\right)^{2} .
$$

This is the set of PDEs we aim to solve for the unknowns $p(\zeta, \bar{\zeta}), c(\zeta, \bar{\zeta})$ and $n(\zeta, \bar{\zeta})$.

Generalizing the ansatz [14, 17] we found from the classical symmetries [21] of the type $\mathrm{N}$ case of (38,41) with $n=0$, we assume the following forms for the unknowns:

$p(\zeta, \bar{\zeta})=\frac{F_{1}(z)}{\sqrt{A \bar{A}}}, \quad c(\zeta, \bar{\zeta})=\frac{\partial_{\zeta} A+\mathrm{i} F_{2}(z)+C_{1}}{A}, \quad n(\zeta, \bar{\zeta})=\frac{F_{3}(z)+\mathrm{i} F_{4}(z)}{(A \bar{A})^{3}}$

with a new real variable

$$
z=-\mathrm{i}\left(\int \frac{1}{A} \mathrm{~d} \zeta-\int \frac{1}{\bar{A}} \mathrm{~d} \bar{\zeta}\right)=\operatorname{Im} \int \frac{2}{A} \mathrm{~d} \zeta .
$$

Here the function $A=A(\zeta)$ is an arbitrary function of $\zeta$ that is sufficiently smooth, and the constant $C_{1}$ and the undetermined functions $F_{1-4}(z)$ are all real-valued. The constraint equation (38) has been taken into account in the form of $c(\zeta, \bar{\zeta})$ so that it is satisfied.

Inserting the ansatz (42) into (39, 41), we obtain a remarkable reduction to a system of four compatible real ODEs for $F_{1-4}(z)$ only, with all other dependence on $A, \bar{A} \neq 0$ factored out:

$$
\begin{aligned}
& 0=-F_{1}^{\prime \prime}+F_{2} F_{1}^{\prime}+\frac{1}{3} \Lambda F_{1}^{3}+\frac{1}{4}\left(3 F_{2}^{\prime}-F_{2}^{2}-C_{1}^{2}\right) F_{1}+\frac{F_{3}}{F_{1}^{3}}, \\
& 0=F_{3}^{\prime}-3\left(F_{2} F_{3}+C_{1} F_{4}\right), \\
& 0=F_{4}^{\prime}+3\left(C_{1} F_{3}-F_{2} F_{4}\right), \\
& 0=\left(H^{\prime}-2 F_{2} H\right)^{\prime}-2 F_{2}\left(H^{\prime}-2 F_{2} H\right)+4 C_{1}^{2} H,
\end{aligned}
$$

where the function $H(z)$ is defined by

$$
H=F_{1}^{\prime \prime} F_{1}-\left(F_{1}^{\prime}\right)^{2}-\Lambda F_{1}^{4}-F_{2}^{\prime} F_{1}^{2} .
$$

The two inner equations are derived from the real and imaginary parts of (40), respectively. Note that they are linear in $F_{3}$ and $F_{4}$. Thus if $F_{2}$ is given, one can solve them by

$$
\begin{aligned}
& F_{3}=\exp \left(3 \int F_{2} \mathrm{~d} z\right)\left[B_{1} \sin \left(3 C_{1} z\right)-B_{2} \cos \left(3 C_{1} z\right)\right] \\
& F_{4}=\exp \left(3 \int F_{2} \mathrm{~d} z\right)\left[B_{2} \sin \left(3 C_{1} z\right)+B_{1} \cos \left(3 C_{1} z\right)\right]
\end{aligned}
$$


where $B_{1,2}$ are real constants. With $F_{3}$ expressed in terms of $F_{2}$, we are left with only two nonlinear equations for $F_{1}$ and $F_{2}$ :

$$
\begin{aligned}
0= & -F_{1}^{\prime \prime}+F_{2} F_{1}^{\prime}+\frac{1}{3} \Lambda F_{1}^{3}+\frac{1}{4}\left(3 F_{2}^{\prime}-F_{2}^{2}-C_{1}^{2}\right) F_{1} \\
& +\frac{\exp \left(3 \int F_{2} \mathrm{~d} z\right)}{F_{1}^{3}}\left[B_{1} \sin \left(3 C_{1} z\right)-B_{2} \cos \left(3 C_{1} z\right)\right], \\
0= & \left(H^{\prime}-2 F_{2} H\right)^{\prime}-2 F_{2}\left(H^{\prime}-2 F_{2} H\right)+4 C_{1}^{2} H,
\end{aligned}
$$

which can be easily converted to a set of ODEs if one introduces, e.g., $F_{2}=K^{\prime}(z)$ to remove the integral in the first equation. The system (48) with (47), or alternatively (43-46), constitutes the main result of this paper.

Before moving on to solve (43,46) in the next few sections, we should make a few general remarks concerning the metric (120) equipped with the ansatz (42). First, despite the appearance of a free function $A(\zeta) \neq 0$ in the ansatz, its different choices do not generate new metrics. In fact, using the coordinate change $\zeta^{\prime}=\int \frac{2}{A(\zeta)} \mathrm{d} \zeta$ permitted by (21) and, accordingly, the transformation law (24) with $f^{\prime}=2 / A(\zeta)$, one can always replace a function $A$ by a constant $A=2$. This is also consistent with the fact that the local CR structure determined by the function $c$ in (42) is independent of the choice of $A(\zeta)$ [14]. Hence for simplicity, we can just set $A(\zeta)=\bar{A}(\bar{\zeta})=2$ without loss of generality, and hence $z=\operatorname{Im} \zeta=y$. With this choice of $A$, the function $L$ and the 1 -form $\lambda$ can be determined from (20) and (18) as

$$
\begin{aligned}
L & =-\mathrm{e}^{-C_{1} x} \int \exp \left(\int F_{2} \mathrm{~d} z\right) \mathrm{d} z, \\
\lambda & =\frac{\mathrm{e}^{C_{1} x} \mathrm{~d} u-2\left[\int \exp \left(\int F_{2} \mathrm{~d} z\right) \mathrm{d} z\right] \mathrm{d} x}{\exp \left(\int F_{2} \mathrm{~d} z\right)} .
\end{aligned}
$$

Once again, though there exist other $L$ 's satisfying (20), one can always use the remaining coordinate freedom $u^{\prime}=F(\zeta, \bar{\zeta}, u$ ) (cf. (21)) to convert them to the $u$ independent real expression (49) [14]. Given such $L$ and $\lambda$, the class of metrics determined by (42,46) admits at least two Killing vectors

$$
X_{1}=\partial_{u}, \quad X_{2}=\partial_{x}-C_{1} u \partial_{u}
$$

with the commutation relation

$$
\left[X_{1}, X_{2}\right]=-C_{1} X_{1}
$$

These vectors, verifiable by direct calculation, are both inherited from the symmetries of the underlying CR structures [22, 17].

\section{Type D solutions: Kerr-NUT}

All type D vacuum solutions, twisting or not, are known [23, 24, which include perhaps the most famous algebraically special solutions such as Kerr's rotating blackhole solution. Hence it is worthwhile to consider whether our equations (43,46) can capture some of these physically important solutions. But first we should comment 
that the CR formalism (11-20) used here is constructed on just one single shearfree null congruence aligned with a multiple principal null direction, which is unique in type II, III and N spacetimes. However, a type D spacetime possesses two such congruences, each along one of the two doubly degenerate principal null directions, and consequently one cannot treat them at the same time in the CR formalism. This suggests that the CR formalism may not provide the most convenient approach for finding type D solutions, as compared to other approaches that are specially designed to make use of both congruences.

A spacetime is of type $\mathrm{D}$ iff it satisfies the conditions

$$
3 \Psi_{3} \Psi_{4}-2 \Psi_{2}^{2}=0, \quad \Psi_{2} \neq 0 .
$$

This equality with the ansatz (42) applied gives rise to a number of lengthy ODEs (as one may sense by looking at the expression of $\Psi_{4}$ ) from the coefficients of various powers of $\mathrm{e}^{\mathrm{i} r}$ required to vanish. This fairly complicated situation (except when $\Psi_{3}=\Psi_{4}=0$, see Appendix A needs a specialized paper to elaborate; hence it is not further discussed here (also because there is no new type D solution to be found). Instead, by applying the results of Section 3, we will simply show that the Kerr-NUT solution can be retrieved as a special solution of (43,46) through the ansatz (42).

In the canonical frame (26-31), the Kerr-NUT solution [12] (p. 453) is given by

$$
\begin{aligned}
& P_{s}=1+\frac{\zeta \bar{\zeta}}{2}, \quad L=-\frac{\mathrm{i}}{\zeta P_{s}^{2}}[2 M+(M+a) \zeta \bar{\zeta}], \\
& m_{s}=m, \quad M_{s}=M, \quad \Lambda=0,
\end{aligned}
$$

where $m, M$ and $a$, each a real constant, are called the mass, the NUT parameter and the Kerr parameter, respectively. Then inserting them into (35), (20) and (37), we obtain the following solution to (38,41):

$$
\begin{aligned}
p & =-\frac{4(M-a)+2(M+a) \zeta \bar{\zeta}}{(2+\zeta \bar{\zeta})^{2}}, \\
c & =\frac{2 \bar{\zeta}[2(M-2 a)+(M+a) \zeta \bar{\zeta}]}{[2(M-a)+(M+a) \zeta \bar{\zeta}](2+\zeta \bar{\zeta})}, \\
n & =-\frac{16 \mathrm{i}[2(M-a)+(M+a) \zeta \bar{\zeta}]^{3}(m+\mathrm{i} M)}{(2+\zeta \bar{\zeta})^{9}} .
\end{aligned}
$$

which, as expected, satisfies the condition (52) for type $\mathrm{D}$ with nonzero $\Psi_{3}$ and $\Psi_{4}$. Without a dependence on $u$, these expressions can be cast into the form of our ansatz (42) by

$$
A(\zeta)=-\mathrm{i} \zeta, \quad z=\log (\zeta \bar{\zeta}), \quad C_{1}=0
$$

such that

$$
\begin{aligned}
& F_{1}=-\frac{2\left[2(M-a)+(M+a) \mathrm{e}^{z}\right] \mathrm{e}^{z / 2}}{\left(2+\mathrm{e}^{z}\right)^{2}}, \quad F_{2}=\frac{4(M-a)+8 a \mathrm{e}^{z}-(M+a) \mathrm{e}^{2 z}}{\left[2(M-a)+(M+a) \mathrm{e}^{z}\right]\left(2+\mathrm{e}^{z}\right)} \\
& F_{3}=\frac{16 M\left[2(M-a)+(M+a) \mathrm{e}^{z}\right]^{3} \mathrm{e}^{3 z}}{\left(2+\mathrm{e}^{z}\right)^{9}}, \quad F_{4}=-\frac{16 m\left[2(M-a)+(M+a) \mathrm{e}^{z}\right]^{3} \mathrm{e}^{3 z}}{\left(2+\mathrm{e}^{z}\right)^{9}}
\end{aligned}
$$


One can verify that they are indeed a solution to (43,46) with $\Lambda=0$. Note that the function $A(\zeta)$ above will still be serving as a free function, as long as $F_{1-4}$ are obtained. The Kerr-NUT solution is contained in the Demiański solution as a special case (cf. (68)). For other examples of type D solutions, see Appendix B and (69) with $B_{2}=0$.

\section{Type $\mathrm{N}$ solutions with nonzero cosmological constant}

The type $\mathrm{N}$ solutions require

$$
\Psi_{2}=\Psi_{3}=0, \quad \Psi_{4} \neq 0 .
$$

These conditions lead to the following special case of (43,46) with $F_{3}=F_{4}=0$ :

$$
\begin{aligned}
& 0=-F_{1}^{\prime \prime}+F_{2} F_{1}^{\prime}+\frac{1}{3} \Lambda F_{1}^{3}+\frac{1}{4}\left(3 F_{2}^{\prime}-F_{2}^{2}-C_{1}^{2}\right) F_{1}, \\
& \Psi_{3} \propto H^{\prime}-2 F_{2} H-2 \mathrm{i} C_{1} H=0,
\end{aligned}
$$

plus one inequality

$$
\begin{aligned}
\Psi_{4}= & -\frac{4 \Lambda}{3 \bar{A}^{2} F_{1}^{2}}\left[2 F_{1} F_{1}^{\prime \prime}+6\left(F_{1}^{\prime}\right)^{2}-10\left(F_{2}+\mathrm{i} C_{1}\right) F_{1} F_{1}^{\prime}\right. \\
& \left.-\left(F_{2}^{\prime}-3 F_{2}^{2}-6 \mathrm{i} C_{1} F_{2}+3 C_{1}^{2}\right) F_{1}^{2}\right] \mathrm{e}^{-\mathrm{i} r / 2} \cos ^{3}\left(\frac{r}{2}\right) \neq 0,
\end{aligned}
$$

which imposes $\Lambda \neq 0$ for type $N$. To better see that the system (54) is included in (43-46), one can rewrite equation (46) as

$$
0=\left(H^{\prime}-2 F_{2} H-2 \mathrm{i} C_{1} H\right)^{\prime}-2\left(F_{2}^{\prime}-\mathrm{i} C_{1}\right)\left(H^{\prime}-2 F_{2} H-2 \mathrm{i} C_{1} H\right) .
$$

In fact, the field equation $R_{33}=0$ can always be removed by $\Psi_{2}=\Psi_{3}=0$ for general type $\mathrm{N}$ vacuums (cf. (15, 17) ). Notice that the second equation of (54) is complex; we have two cases for solutions.

Case 1: $H=0, \Lambda \neq 0$. This simpler case has been investigated in [14] (see [17] for more details). The equations for this case read

$$
\begin{aligned}
& 0=-F_{1}^{\prime \prime}+F_{2} F_{1}^{\prime}+\frac{1}{3} \Lambda F_{1}^{3}+\frac{1}{4}\left(3 F_{2}^{\prime}-F_{2}^{2}-C_{1}^{2}\right) F_{1}, \\
& 0=H=-F_{1}^{\prime \prime} F_{1}+\left(F_{1}^{\prime}\right)^{2}+\Lambda F_{1}^{4}+F_{2}^{\prime} F_{1}^{2} .
\end{aligned}
$$

By introducing a real function $J=J(z)$ and

$$
F_{1}= \pm \sqrt{J^{\prime}}, \quad F_{2}=\frac{J^{\prime \prime}}{2 J^{\prime}}-\Lambda J, \quad J^{\prime}>0
$$

we can reduce the first equation of (55) to

$$
J^{\prime \prime \prime}=\frac{\left(J^{\prime \prime}\right)^{2}}{2 J^{\prime}}-2 \Lambda J J^{\prime \prime}-\frac{10}{3} \Lambda\left(J^{\prime}\right)^{2}-2\left(\Lambda^{2} J^{2}+C_{1}^{2}\right) J^{\prime}
$$

while the second equation is automatically satisfied. Since this ODE has no explicit dependence on the variable $z$, we can immediately lower its order by the transformation $J^{\prime}=P(J)>0$ such that

$$
P^{\prime \prime}=-\frac{\left(P^{\prime}+2 \Lambda J\right)^{2}}{2 P}-\frac{2 C_{1}^{2}}{P}-\frac{10}{3} \Lambda, \quad \Lambda \neq 0
$$


which, in the case of $C_{1}=0$, can be further reduced to an Abel ODE [25] of the first kind

$$
f^{\prime}=\frac{4}{t}\left(t+\frac{3}{2}\right)\left(t+\frac{1}{3}\right) f^{3}+\frac{5}{t}\left(t+\frac{2}{5}\right) f^{2}+\frac{1}{2 t} f,
$$

by $J=\exp \left(\int f(t) \mathrm{d} t\right) / \Lambda, P(J)=t \exp \left(2 \int f(t) \mathrm{d} t\right) / \Lambda$. Unfortunately, this Abel ODE has not been identified as a known solvable type.

Various aspects of the equation (58) were examined in [14], including the weak Painlevé property [26] and constructions of various special and series solutions. All degenerate solutions of type $\mathrm{O}\left(\Psi_{4}=0\right.$, conformally flat $)$ were found. The only known type $\mathrm{N}$ solution with $\Lambda \neq 0$ in closed forms was first discovered by Leroy [27] and presented in the CR formalism by Nurowski [15]. It corresponds to

$$
\begin{aligned}
& P(J)=-\frac{1}{3} \Lambda J^{2}-\frac{3 C_{1}^{2}}{4 \Lambda}>0, \\
& F_{1}= \pm \frac{\sqrt{3} C_{1}}{2 s \sin \left(\frac{1}{2} C_{1}\left(z+C_{0}\right)\right)}, \quad F_{2}=-\frac{2 C_{1}}{\tan \left(\frac{1}{2} C_{1}\left(z+C_{0}\right)\right)},
\end{aligned}
$$

with $\Lambda=-s^{2}<0$ and $C_{0}$ a real constant (removable by a translation $z+C_{0} \rightarrow z$ ). Nonetheless, the equation (58) does also admit type $N$ solutions with $\Lambda>0$ and two additional parameters besides $\Lambda$ and $C_{1}$.

Case 2: $H \neq 0, C_{1}=0, \Lambda \neq 0$. The system (54) becomes

$$
\begin{aligned}
& 0=-F_{1}^{\prime \prime}+F_{2} F_{1}^{\prime}+\frac{1}{3} \Lambda F_{1}^{3}+\frac{1}{4}\left(3 F_{2}^{\prime}-F_{2}^{2}\right) F_{1}, \\
& 0=H^{\prime}-2 F_{2} H .
\end{aligned}
$$

Very little is known about the solutions of this system except for one of type $\mathrm{O}$ [17] given by

$$
F_{1}= \pm \frac{\sqrt{6}}{2 s\left(z+C_{0}\right)}, \quad F_{2}=-\frac{2}{z+C_{0}}, \quad H=\frac{3}{4 s^{2}\left(z+C_{0}\right)^{4}},
$$

with $\Lambda=-s^{2}<0$ and $C_{0}$ a real constant. Particularly, this solution has the hyperquadric CR structure (the most symmetrical one) [28].

\section{Type III solutions}

Similar to the case of type $\mathrm{N}$, the equations for type III $\left(\Psi_{2}=0, \Psi_{3} \neq 0 ; F_{3}=F_{4}=0\right)$ are given by

$$
\begin{aligned}
& 0=-F_{1}^{\prime \prime}+F_{2} F_{1}^{\prime}+\frac{1}{3} \Lambda F_{1}^{3}+\frac{1}{4}\left(3 F_{2}^{\prime}-F_{2}^{2}-C_{1}^{2}\right) F_{1}, \\
& 0=\left(H^{\prime}-2 F_{2} H\right)^{\prime}-2 F_{2}\left(H^{\prime}-2 F_{2} H\right)+4 C_{1}^{2} H,
\end{aligned}
$$

which are subject to

$$
\Psi_{3} \propto H^{\prime}-2 F_{2} H-2 \mathrm{i} C_{1} H \neq 0 .
$$

Using the first equation of (63), we can lower the order of the second ODE, such that the resulting set of equations contains derivatives up to the second-order in $F_{1}$ and the third-order in $F_{2}$. Hence the general solution carries another five real parameters in 
addition to $\Lambda$ and $C_{1}$. Considering that one of these parameters is simply the translation $z \rightarrow z+C_{0}$ (no explicit dependence on $z$ in (43,46) ), and thus removable, one can see that the final type III metric determined by (63) has at most six parameters including $\Lambda$ and $C_{1}$ (see the conclusions).

Two classes of twisting type III vacuum solutions are known, respectively for $\Lambda=0$ and $\Lambda<0$. The one with $\Lambda=0$ is due to Held [29] and Robinson [30], which generalizes the non-twisting Robinsion-Trautman type III vacuum solution and generally admits only one Killing vector $\partial_{u}$. The subclasses with two Killing vectors (commuting or not) were found by Lun [31]. It can be shown that Lun's case I type III metric corresponds to the following solution of (63):

$$
\begin{aligned}
& \Lambda=0, \quad C_{1}=0, \\
& F_{1}=\frac{\sqrt{3}}{2 z^{3}}\left(E_{1} z^{2+\sqrt{13} / 2}+E_{2} z^{2-\sqrt{13} / 2}\right), \\
& F_{2}=-\frac{(5-\sqrt{13}) E_{1} z^{2+\sqrt{13} / 2}+(5+\sqrt{13}) E_{2} z^{2-\sqrt{13} / 2}}{2 z\left(E_{1} z^{2+\sqrt{13} / 2}+E_{2} z^{2-\sqrt{13}} / 2\right)} .
\end{aligned}
$$

Likewise, his case II type III metric puts forth a second solution of (63):

$$
\begin{aligned}
& \Lambda=0, \quad C_{1}=\frac{1}{4}, \\
& F_{1}=\frac{\sqrt{3}}{4 \cos \left(\frac{z}{2}\right)}\left(E_{1} G+E_{2} G^{-1}\right), \\
& F_{2}=\frac{\sqrt{13}\left(E_{1} G-E_{2} G^{-1}\right)}{4 \cos \left(\frac{z}{2}\right)\left(E_{1} G+E_{2} G^{-1}\right)}+\frac{5}{4} \tan \left(\frac{z}{2}\right), \\
& G(z)=\left(\frac{\sin \left(\frac{z}{2}\right)+1}{\cos \left(\frac{z}{2}\right)}\right)^{\sqrt{13} / 2} .
\end{aligned}
$$

In both cases, $E_{1,2}$ are real constants. More details will be given in the next section as degenerate cases of the related type II solutions (67) and (70).

The other known type III solution requires $\Lambda<0$ and is due to MacCallum and Siklos [32] (see also [12] p. 201). As a solution of (63), it is given by

$$
F_{1}= \pm \frac{\sqrt{39}}{4 s\left(z+C_{0}\right)}, \quad F_{2}=-\frac{5}{2\left(z+C_{0}\right)}, \quad C_{1}=0,
$$

with $\Lambda=-s^{2}$, a real constant $C_{0}$ and

$$
\Psi_{3} \propto H^{\prime}-2 F_{2} H=-\frac{585}{256 \Lambda\left(z+C_{0}\right)^{5}} .
$$

\section{Type II solutions}

Based on the structure of (43,46), we can consider the type II solution $\left(\Psi_{2} \neq 0\right.$, $3 \Psi_{2} \Psi_{4}-2 \Psi_{3}^{2} \neq 0$ ) according to three different cases. 
Case 1: $F_{3}=0, F_{4} \neq 0 \Rightarrow C_{1}=0$. The equations (43,46) are reduced to

$$
\begin{aligned}
& 0=-F_{1}^{\prime \prime}+F_{2} F_{1}^{\prime}+\frac{1}{3} \Lambda F_{1}^{3}+\frac{1}{4}\left(3 F_{2}^{\prime}-F_{2}^{2}\right) F_{1}, \\
& 0=\left(H^{\prime}-2 F_{2} H\right)^{\prime}-2 F_{2}\left(H^{\prime}-2 F_{2} H\right), \\
& F_{4}=B_{1} \exp \left(3 \int F_{2} \mathrm{~d} z\right),
\end{aligned}
$$

with $B_{1} \neq 0$ a real constant. Since the first two equations above are identical to (63) with $C_{1}=0$ (also cf. (55) and (61) ), one can generate this kind of type II solutions directly from existing type $\mathrm{N}$ and III solutions, i.e., (60) with $C_{1}=0$, (64) and (66), which works as if one is adding a "mass source" to them. A similar idea can be found in [12] p. 447. In addition, for type $\mathrm{D}$ solutions with $F_{3}=0$ and $C_{1}=0$, see Appendix B.

Case 2: $F_{3} \neq 0, C_{1}=0$. The associated equations are given by

$$
\begin{aligned}
& 0=-F_{1}^{\prime \prime}+F_{2} F_{1}^{\prime}+\frac{1}{3} \Lambda F_{1}^{3}+\frac{1}{4}\left(3 F_{2}^{\prime}-F_{2}^{2}\right) F_{1}+\frac{F_{3}}{F_{1}^{3}}, \\
& 0=\left(H^{\prime}-2 F_{2} H\right)^{\prime}-2 F_{2}\left(H^{\prime}-2 F_{2} H\right), \\
& F_{2}=\frac{F_{3}^{\prime}}{3 F_{3}}, \quad F_{4}=B_{1} \exp \left(3 \int F_{2} \mathrm{~d} z\right)=B_{1} F_{3},
\end{aligned}
$$

with $B_{1}$ a real constant. Certainly one may use the third equation above to turn the first two into ODEs for $F_{1}$ and $F_{3}$ only.

Lun's case I solution [31, 33] with four parameters can be shown to belong to this case. It reads, in the canonical frame (26-31),

$$
\begin{aligned}
& P_{s}=\sqrt{\frac{2}{3}}(\zeta+\bar{\zeta})^{3 / 2}, \quad \zeta=x+\mathrm{i} y, \\
& L=-\frac{3 \mathrm{i}}{16} x^{-3 / 2}\left[(3+\sqrt{13}) E_{1} x^{\sqrt{13} / 2}+(3-\sqrt{13}) E_{2} x^{-\sqrt{13} / 2}\right]+\frac{3 \mathrm{i} M}{32 x^{3}}, \\
& m_{s}=m, \quad M_{s}=M, \quad \Lambda=0,
\end{aligned}
$$

or, in the CR formalism as a solution of (38, 41) or (43,46),

$$
\begin{aligned}
& A(\zeta)=-2 \mathrm{i}, \quad z=x, \quad C_{1}=0, \\
& p=\frac{1}{2} F_{1}(z)=\frac{\sqrt{3}}{16 z^{3}} G(z) \text {, } \\
& c=-\frac{1}{2} F_{2}(z) \\
& =(z G)^{-1}\left[(5-\sqrt{13}) E_{1} z^{2+\sqrt{13} / 2}+(5+\sqrt{13}) E_{2} z^{2-\sqrt{13} / 2}+6 M z^{1 / 2}\right], \\
& n=\frac{1}{64}\left(F_{3}(z)+\mathrm{i} F_{4}(z)\right)=\frac{27 \mathrm{i}}{2^{20} z^{27 / 2}} G^{3}(m+\mathrm{i} M), \\
& G(z)=4\left(E_{1} z^{2+\sqrt{13} / 2}+E_{2} z^{2-\sqrt{13} / 2}\right)+3 M z^{1 / 2},
\end{aligned}
$$

with $m, M$ and $E_{1,2}$ real constants. When $m=M=0$, the solution degenerates to the type III solution (64).

The Kerr and Debney/Demiański's four-parameter solution [12] (see p. 449) also 
falls under this case. It is given by

$$
\begin{aligned}
& P_{s}=1+\frac{\zeta \bar{\zeta}}{2}, \quad L=-\mathrm{i} P_{s}^{2}\left[2 M / \zeta+(M+a) \bar{\zeta}+\frac{1}{4} b \bar{\zeta} \log (\bar{\zeta} / \sqrt{2})\right], \\
& m_{s}=m, \quad M_{s}=M, \quad \Lambda=0
\end{aligned}
$$

and corresponds to

$$
\begin{aligned}
& A(\zeta)=-\mathrm{i} \zeta, \quad z=\log (\zeta \bar{\zeta}), \quad C_{1}=0, \\
& F_{1}=-\frac{\left[16(M-a)+8(M+a) \mathrm{e}^{z}+b G_{1}\right] \mathrm{e}^{z / 2}}{4\left(2+\mathrm{e}^{z}\right)^{2}}, \\
& F_{2}=\frac{8\left[4(M-a)+8 a \mathrm{e}^{z}-(M+a) \mathrm{e}^{2 z}\right]+b G_{2}}{8\left[2(M-a)+(M+a) \mathrm{e}^{z}\right]\left(2+\mathrm{e}^{z}\right)+b G_{1}\left(2+\mathrm{e}^{z}\right)}, \\
& F_{3}=\frac{M\left[16(M-a)+8(M+a) \mathrm{e}^{z}+b G_{1}\right]^{3} \mathrm{e}^{3 z}}{32\left(2+\mathrm{e}^{z}\right)^{9}}, \\
& F_{4}=-\frac{m\left[16(M-a)+8(M+a) \mathrm{e}^{z}+b G_{1}\right]^{3} \mathrm{e}^{3 z}}{32\left(2+\mathrm{e}^{z}\right)^{9}}, \\
& G_{1}(z)=(2+\ln 2-z)\left(2-\mathrm{e}^{z}\right)-8 \\
& G_{2}(z)=(3+\ln 2-z)\left(4+\mathrm{e}^{2 z}\right)-8(\ln 2-z) \mathrm{e}^{z}-24
\end{aligned}
$$

which is a solution of (43,46). Here $m, M, a$ and $b$ are four real parameters. Clearly, the Kerr-NUT solution (53) is a special case with $b=0$.

Besides these known solutions, we have obtained an additional one (see the derivation in Case 3 below) which turns out to be a limiting case $\left(C_{1} \rightarrow 0\right)$ of the solution (72):

$$
\begin{aligned}
& C_{1}=0, \quad \Lambda=0, \\
& F_{1}=-2 B_{2} z^{2}+C_{2} z+C_{3}, \quad F_{2}=\frac{4 B_{2} z-C_{2}}{2 B_{2} z^{2}-C_{2} z-C_{3}}, \\
& F_{3}=-B_{2} F_{1}^{3}, \quad F_{4}=B_{1} F_{1}^{3} .
\end{aligned}
$$

Here $C_{2,3}$ and $B_{1,2}$ are real constants. Its comparisons with Lun's and Demiański's solutions will be discussed in Appendix C. Particularly when $B_{2}=0$, the solution becomes type D.

Case 3: $F_{3} \neq 0, C_{1} \neq 0 \Rightarrow F_{4} \neq 0$. This corresponds to the most general case for solutions. As one may check, Lun's case II four-parameter solution [31, 33], which is given by

$$
\begin{aligned}
& P_{s}=\sqrt{\frac{2}{3}}(\zeta+\bar{\zeta})^{3 / 2}, \quad \zeta=x+\mathrm{i} y, \quad w=y / x, \\
& L=x^{-3 / 2}\left\{\frac { 1 } { 6 } \left[E_{1}\left(w+\left(w^{2}+1\right)^{1 / 2}\right)^{\sqrt{13} / 2}\left(w-\frac{\sqrt{13}}{2}\left(w^{2}+1\right)^{1 / 2}\right)\right.\right. \\
& \left.+E_{2}\left(w+\left(w^{2}+1\right)^{1 / 2}\right)^{-\sqrt{13} / 2}\left(w+\frac{\sqrt{13}}{2}\left(w^{2}+1\right)^{1 / 2}\right)\right] \\
& \left.+\frac{3}{160}\left[(m+\mathrm{i} M)(1+\mathrm{i} w)^{3 / 2}(2-3 \mathrm{i} w)+(m-\mathrm{i} M)(1-\mathrm{i} w)^{3 / 2}(2+3 \mathrm{i} w)\right]\right\}, \\
& m_{s}-\mathrm{i} M_{s}=(m+\mathrm{i} M) x^{3 / 2}(1+\mathrm{i} w)^{3 / 2}, \quad \Lambda=0,
\end{aligned}
$$


can be converted to a solution of (43,46):

$$
\begin{aligned}
& A(\zeta)=\zeta, \quad \zeta=|\zeta| \mathrm{e}^{\mathrm{i} z / 2}, \quad C_{1}=\frac{1}{4}, \\
& F_{1}=\frac{\sqrt{3}\left(E_{1} G+E_{2} G^{-1}\right)}{4 \cos \left(\frac{z}{2}\right)}+\frac{3 \sqrt{3} \sin \left(\frac{z}{2}\right)\left(M \sin \left(\frac{z}{4}\right)-m \cos \left(\frac{z}{4}\right)\right)}{16 \cos ^{5 / 2}\left(\frac{z}{2}\right)}, \\
& F_{2}=\frac{4 \cos ^{3 / 2}\left(\frac{z}{2}\right)\left[5 \sin \left(\frac{z}{2}\right)\left(E_{1} G+E_{2} G^{-1}\right)+\sqrt{13}\left(E_{1} G-E_{2} G^{-1}\right)\right]-3\left(M G_{1}-m G_{2}\right)}{16 \cos ^{5 / 2}\left(\frac{z}{2}\right)\left(E_{1} G+E_{2} G^{-1}\right)+6 \sin (z)\left(M \sin \left(\frac{z}{4}\right)-m \cos \left(\frac{z}{4}\right)\right)}, \\
& F_{3}=\frac{3 \sqrt{3} F_{1}^{3}\left(M \cos \left(\frac{3 z}{4}\right)+m \sin \left(\frac{3 z}{4}\right)\right)}{2^{8} \cos ^{9 / 2}\left(\frac{z}{2}\right)}, \quad F_{4}=\frac{3 \sqrt{3} F_{1}^{3}\left(-M \sin \left(\frac{3 z}{4}\right)+m \cos \left(\frac{3 z}{4}\right)\right)}{2^{8} \cos ^{9 / 2}\left(\frac{z}{2}\right)}, \\
& G(z)=\left(\frac{\sin \left(\frac{z}{2}\right)+1}{\cos \left(\frac{z}{2}\right)}\right)^{\sqrt{13} / 2}, \quad G_{1}(z)=\frac{5}{4} \sin \left(\frac{5 z}{4}\right)-\frac{7}{4} \sin \left(\frac{3 z}{4}\right)-5 \sin \left(\frac{z}{4}\right), \\
& G_{2}(z)=\frac{5}{4} \cos \left(\frac{5 z}{4}\right)+\frac{7}{4} \cos \left(\frac{3 z}{4}\right)-5 \cos \left(\frac{z}{4}\right),
\end{aligned}
$$

with $E_{1,2}, M$ and $m$ real constants. In the case of $M=m=0$, the solution reduces to the type III solution (65).

Besides Lun's example, we have also considered the special case of $H=0$ for the system (43,46), which turns out to be fully soluble when $\Lambda=0$. The derivation follows closely the type N Case 1 (cf. (55)), and utilizes the same ansatz (56) that makes the function $H(z)$ vanish (hence, (46) satisfied). More specifically, we have

$$
\begin{aligned}
& F_{1}= \pm \sqrt{J^{\prime}}, \quad F_{2}=\frac{J^{\prime \prime}}{2 J^{\prime}}-\Lambda J=\frac{F_{1}^{\prime}}{F_{1}}-\Lambda J, \quad J^{\prime}>0, \\
& F_{3}=F_{1}^{3} \exp \left(-3 \Lambda \int J \mathrm{~d} z\right)\left[B_{1} \sin \left(3 C_{1} z\right)-B_{2} \cos \left(3 C_{1} z\right)\right], \\
& F_{4}=F_{1}^{3} \exp \left(-3 \Lambda \int J \mathrm{~d} z\right)\left[B_{2} \sin \left(3 C_{1} z\right)+B_{1} \cos \left(3 C_{1} z\right)\right],
\end{aligned}
$$

with the last two equations derived from (47). Therefore when $\Lambda$ vanishes, the equation (43) can be reduced to a linear ODE for $F_{1}(z)$ alone:

$$
F_{1}^{\prime \prime}=-C_{1}^{2} F_{1}+4\left[B_{1} \sin \left(3 C_{1} z\right)-B_{2} \cos \left(3 C_{1} z\right)\right], \quad \Lambda=0,
$$

which has the general solution

$$
F_{1}=C_{2} \cos \left(C_{1}\left(z+C_{0}\right)\right)-\frac{1}{2 C_{1}^{2}}\left[B_{1} \sin \left(3 C_{1} z\right)-B_{2} \cos \left(3 C_{1} z\right)\right], \quad C_{1} \neq 0,
$$

or, if $C_{1}$ vanishes (cf. (69) ),

$$
F_{1}=-2 B_{2} z^{2}+C_{3} z+C_{4}, \quad C_{1}=0,
$$

where $C_{0-4}$ and $B_{1,2}$ are real constants. The conditions $H=0$ and $\Lambda=0$ exclude type III and $\mathrm{N}$ as special cases. The solution corresponding to (72) with $C_{1}=-\frac{1}{2}$ coincides with a special case of Kerr and Debney's type II solution [12] (p. 608), and hence is not new (see Appendix D for more details). Also, by setting $C_{0}=0, C_{2}=-\frac{B_{2}}{2 C_{1}^{2}}+C_{4}$ and $B_{1}=-\frac{2}{3} C_{1} C_{3}$, one can obtain (73) from (72) in the limit $C_{1} \rightarrow 0$, whereas the two 
Killing vectors (51) become commuting. For a generally non-vanishing $\Lambda$, we obtain from (43) an equation for $J(z)$ :

$$
\begin{aligned}
J^{\prime \prime \prime} & =\frac{\left(J^{\prime \prime}\right)^{2}}{2 J^{\prime}}-2 \Lambda J J^{\prime \prime}-\frac{10}{3} \Lambda\left(J^{\prime}\right)^{2}-2\left(\Lambda^{2} J^{2}+C_{1}^{2}\right) J^{\prime} \\
& +8\left(J^{\prime}\right)^{1 / 2} \exp \left(-3 \Lambda \int J \mathrm{~d} z\right)\left[B_{1} \sin \left(3 C_{1} z\right)-B_{2} \cos \left(3 C_{1} z\right)\right],
\end{aligned}
$$

which generalizes the equation (57) for type II. By introducing $J=K^{\prime}$ (or $-3 \Lambda J=$ $K^{\prime} / K$, etc.), one can transform this equation to a fourth-order ODE for $K(z)$. However, we do not have any type II solution with $\Lambda \neq 0$ for this ODE.

\section{Conclusions}

With the real coordinates $\{x, z, u, r\}$ and $A(\zeta)=2$ in the ansatz (42), we present here our new class of vacuum twisting type II metrics admitting two Killing vectors:

$$
\mathbf{g}=\frac{F_{1}^{2}(z)}{2 \cos ^{2}\left(\frac{r}{2}\right)}[\mathrm{d} \zeta \mathrm{d} \bar{\zeta}+\lambda(\mathrm{d} r+\mathcal{W} \mathrm{d} \zeta+\mathcal{W} \mathrm{d} \bar{\zeta}+\mathcal{H} \lambda)]
$$

with $\zeta=x+\mathrm{i} z$ and

$$
\begin{gathered}
\lambda=\frac{\mathrm{e}^{C_{1} x} \mathrm{~d} u-2\left[\int \exp \left(\int F_{2} \mathrm{~d} z\right) \mathrm{d} z\right] \mathrm{d} x}{\exp \left(\int F_{2} \mathrm{~d} z\right)} \\
\mathcal{W}=\frac{1}{2}\left(\frac{2 F_{1}^{\prime}}{F_{1}}-F_{2}+\mathrm{i} C_{1}\right)\left(\mathrm{e}^{-\mathrm{i} r}+1\right), \\
\mathcal{H}=-\frac{1}{2}\left[\left(\frac{F_{1}^{\prime}}{F_{1}}\right)^{\prime}-\Lambda F_{1}^{2}-F_{2}^{\prime}-\frac{2\left(F_{3}(\cos r+1)+F_{4} \sin r\right)}{F_{1}^{4}}\right](\cos r+1)-\frac{1}{6} \Lambda F_{1}^{2} \cos r
\end{gathered}
$$

where $C_{1}$ is an arbitrary real parameter and the real functions $F_{1-4}(z)$ satisfy

$$
\begin{aligned}
& 0=-F_{1}^{\prime \prime}+F_{2} F_{1}^{\prime}+\frac{1}{3} \Lambda F_{1}^{3}+\frac{1}{4}\left(3 F_{2}^{\prime}-F_{2}^{2}-C_{1}^{2}\right) F_{1}+\frac{F_{3}}{F_{1}^{3}}, \\
& 0=\left(H^{\prime}-2 F_{2} H\right)^{\prime}-2 F_{2}\left(H^{\prime}-2 F_{2} H\right)+4 C_{1}^{2} H, \\
& F_{3}=\exp \left(3 \int F_{2} \mathrm{~d} z\right)\left[B_{1} \sin \left(3 C_{1} z\right)-B_{2} \cos \left(3 C_{1} z\right)\right], \\
& F_{4}=\exp \left(3 \int F_{2} \mathrm{~d} z\right)\left[B_{2} \sin \left(3 C_{1} z\right)+B_{1} \cos \left(3 C_{1} z\right)\right],
\end{aligned}
$$

with the function $H(z)$ defined by

$$
H=F_{1}^{\prime \prime} F_{1}-\left(F_{1}^{\prime}\right)^{2}-\Lambda F_{1}^{4}-F_{2}^{\prime} F_{1}^{2} .
$$

One can determine $F_{1,2}$ from the first two equations of (75), which are in fact a pair of ODEs for $F_{1}$ and $K=\int F_{2} \mathrm{~d} z$. This allows the metric to have at most eight real parameters including $\Lambda$ and $C_{1}$ (see discussion in Section 7 ), and its two Killing vectors are

$$
\partial_{u}, \quad \partial_{x}-C_{1} u \partial_{u}
$$


Most of the previously known twisting vacuum solutions with two Killing vectors, as presented in Chapters 29 and 38 of [12], have been shown to belong to this class, the only exception being the general case of Kerr and Debney's type II solution (see Appendix D). Additionally, for $H=0$, the system (75) can be reduced to a single ODE (74), or even be fully integrated when $\Lambda=0$. This leads to the discovery of a limiting solution (69) (type II with two commuting Killing vectors) of a special case of Kerr and Debney's type II solution, which we believe has not been discussed or published before. Despite all these special solutions with maximally four parameters, the general solution of (75) is still quite unknown. We believe that this problem poses a major challenge.

Altogether, we hope that this work may provide a platform for all types of twisting algebraically special solutions to be studied in a connected and unified manner, given the history that many of those known solutions were derived by quite different approaches or special assumptions. For future research, this new class of metrics may be further examined to study important issues such as the cosmic no-hair conjecture, the asymptotic stability of the Kerr solution [34], and the formation of rotating black holes that might be described by certain solutions of (75) (see, e.g., [35, 36] and references therein).

\section{Appendix A. Type D solutions: $\Psi_{3}=\Psi_{4}=0$}

For simplicity, we consider type $\mathrm{D}$ solutions with $\Psi_{2} \neq 0$ and $\Psi_{3}=\Psi_{4}=0$. In order to acquire $\Psi_{3}=0$ with $\Psi_{3}$ depending on $r$, we need, at least, for the coefficient of $\mathrm{e}^{2 \mathrm{i} r}$ to vanish in (17),

$$
0=2 \bar{\partial} \log p+\bar{c}
$$

which, through the ansatz (42), adds two more equations to the system:

$$
C_{1}=0, \quad F_{1}^{\prime}=\frac{1}{2} F_{2} F_{1} .
$$

Thus we can simplify the original (43,46) to

$$
\begin{aligned}
F_{2}^{\prime} & =-\frac{4}{3} \Lambda F_{1}^{2}-\frac{4 F_{3}}{F_{1}^{4}}, \\
F_{3}^{\prime} & =3 F_{2} F_{3}, \\
F_{4}^{\prime} & =3 F_{2} F_{4},
\end{aligned}
$$

with the last equation (46) being automatically satisfied by the equations presented above. The system (A.2 A.5 can be fully solved with the general solution

$$
\begin{gathered}
F_{1}=C_{3} \sec \left(\frac{z+C_{0}}{C_{2}}\right), \quad F_{2}=\frac{2}{C_{2}} \tan \left(\frac{z+C_{0}}{C_{2}}\right), \\
F_{3}=-\frac{C_{3}^{4}\left(3+2 \Lambda C_{2}^{2} C_{3}^{2}\right)}{6 C_{2}^{2}} \sec ^{6}\left(\frac{z+C_{0}}{C_{2}}\right), \quad F_{4}=C_{4} \sec ^{6}\left(\frac{z+C_{0}}{C_{2}}\right) .
\end{gathered}
$$

Remarkably, though we have only started with one extra condition (A.1), it is enough for the solution (A.6) to be of type D, i.e., that we have

$$
\Psi_{2}=\frac{-C_{3}^{4}\left(3+2 \Lambda C_{2}^{2} C_{3}^{2}\right)+6 \mathrm{i} C_{2}^{2} C_{4}}{12 C_{2}^{2} C_{3}^{6}}\left(\mathrm{e}^{\mathrm{i} r}+1\right)^{3}, \quad \Psi_{3}=\Psi_{4}=0 .
$$


With $A(\zeta)=2$ and (A.6), the resulting metric can be written as

$$
\mathbf{g}=\frac{C_{4}^{2}}{2 \cos ^{2}\left(\frac{r}{2}\right) \cos ^{2}\left(\frac{z+C_{0}}{C_{2}}\right)}[\mathrm{d} \zeta \mathrm{d} \bar{\zeta}+\lambda(\mathrm{d} r+\mathcal{H} \lambda)]
$$

with $\zeta=x+\mathrm{i} z$ and

$$
\begin{aligned}
& \lambda=\cos ^{2}\left(\frac{z+C_{0}}{C_{2}}\right)\left[\mathrm{d} u-2 C_{2} \tan \left(\frac{z+C_{0}}{C_{2}}\right) \mathrm{d} x\right], \\
& \mathcal{H}=-\frac{1}{\cos ^{2}\left(\frac{z+C_{0}}{C_{2}}\right)}\left[\cos ^{2}\left(\frac{r}{2}\right)\left(\frac{\cos r}{C_{2}^{2}}+\frac{2 C_{4} \sin r}{C_{3}^{4}}\right)+\frac{1}{6} C_{3}^{2} \Lambda(\cos 2 r+2 \cos r)\right] .
\end{aligned}
$$

One can immediately remove the parameter $C_{0}$ by $z+C_{0} \rightarrow z$. Besides the cosmological constant $\Lambda$, the metric contains three real parameters $C_{2}, C_{3}$ and $C_{4}$.

\section{Appendix B. Type D solutions from classical symmetries}

Here we list a number of special solutions one may encounter when searching for groupinvariant solutions from classical symmetries [21] of the system (43, 46) or its various special cases. Incidentally, all these solutions turn out to be of type D, even though the condition $2 \Psi_{2}^{2}=3 \Psi_{3} \Psi_{4}$ is never used in their derivation. As expected for type D solutions, they all have $C_{1}=0$, which means that the two Killing vectors (51) are commuting. In what follows, $C_{2-4}$ are real constants.

We start with solutions with $F_{3}=0$. For $\Lambda=0$, we have

$$
\begin{aligned}
& F_{1}=C_{3} \mathrm{e}^{C_{2} z}, \quad F_{2}=2 C_{2}, \quad F_{3}=0, \quad F_{4}=C_{4} \mathrm{e}^{6 C_{2} z}, \\
& F_{1}=\frac{C_{2}}{z^{2}}, \quad F_{2}=-\frac{3}{z}, \quad F_{3}=0, \quad F_{4}=\frac{C_{3}}{z^{9}} .
\end{aligned}
$$

For $\Lambda=-s^{2}<0$, we have ([14] and cf. ([62])

$$
\begin{array}{llll}
F_{1}=\frac{\sqrt{6}}{3 s z}, & F_{2}=-\frac{5}{3 z}, & F_{3}=0, & F_{4}=\frac{C_{2}}{z^{5}}, \\
F_{1}=\frac{\sqrt{6}}{2 s z}, & F_{2}=-\frac{2}{z}, & F_{3}=0, & F_{4}=\frac{C_{2}}{z^{6}} .
\end{array}
$$

In case of $F_{4}=0$, they all become type $\mathrm{O}$ solutions of (154) or (63).

For solutions that admit non-vanishing $F_{3}$ and $\Lambda$, we have

$$
\begin{aligned}
& F_{1}=C_{2} \neq 0, \quad F_{2}=0, \quad F_{3}=-\frac{1}{3} C_{2}^{6} \Lambda, \quad F_{4}=C_{3}, \\
& F_{1}=\frac{C_{2}}{z}, \quad F_{2}=-\frac{2}{z}, \quad F_{3}=-\frac{3 C_{2}^{4}+2 C_{2}^{6} \Lambda}{6 z^{6}}, \quad F_{4}=\frac{C_{3}}{z^{6}},
\end{aligned}
$$

Note that the first solution above is given by constants, which is a consequence of the translational invariance $\left(z \rightarrow z+C_{0}\right)$ of the system (43,46). Lastly for $\Lambda=0$, we obtain

$$
F_{1}=C_{3} \mathrm{e}^{C_{2} z}, \quad F_{2}=\frac{4}{3} C_{2}, \quad F_{3}=\frac{1}{9} C_{3}^{4} C_{2}^{2} \mathrm{e}^{4 C_{2} z}, \quad F_{4}=C_{4} \mathrm{e}^{4 C_{2} z} .
$$




\section{Appendix C. Comparisons of type II solutions with $C_{1}=0$}

Here we compare the three type II solutions from Case 2, i.e., (67), (68) and (69), and show that (69) is different from the other two. Generally, to see that two twisting type II vacuum metrics are different, i.e., not being related by a coordinate transformation, it is sufficient to show that their CR structures along the shearfree null congruences are not equivalent [6]. This can be decided by evaluating the six Cartan invariants [5, 14], which are the same only for two equivalent CR structures. Among these invariants, the first one, in Cartan's original notation, is given by

$$
\begin{aligned}
& \alpha(\zeta, \bar{\zeta})=-\frac{5 \bar{r} \partial_{\zeta} r+r \partial_{\zeta} \bar{r}+8 c r \bar{r}}{8 \sqrt{\bar{r}} \cdot \sqrt[8]{(r \bar{r})^{7}}}, \\
& \bar{r}=\frac{1}{6}\left(\partial_{\zeta} l+2 c l\right), \quad l=-\partial_{\zeta} \partial_{\bar{\zeta}} c-c \partial_{\bar{\zeta}} c,
\end{aligned}
$$

which only relies on the function $c=c(\zeta, \bar{\zeta})$ (hence $F_{2}(z)$ from the ansatz (42)). The use of $\alpha$ alone will be adequate for our comparison.

For simplicity, we consider the special case of (69) with $C_{2}=C_{3}=0$, i.e.,

$$
F_{1}=-2 B_{2} z^{2}, \quad F_{2}=\frac{2}{z}, \quad F_{3}=-B_{2} F_{1}^{3}, \quad F_{4}=B_{1} F_{1}^{3} .
$$

This solution is still of type II but has a constant invariant $\alpha$ given by

$$
\alpha^{2}=-\frac{25}{14} \sqrt{21}
$$

Yet another case with $\alpha$ being constant is when $B_{2}=0$ in (69), in which case the solution is of type D and

$$
\alpha^{2}=-\frac{16}{5} \sqrt{10}
$$

This same quantity $\alpha$ calculated from Lun's solution (67), however, is generally a function of $z$ and only becomes a constant when two of the three free parameters $E_{1,2}$ and $M$ vanish, i.e., that we have

$$
\begin{aligned}
& \alpha^{2}=\frac{\sqrt{15}}{10} \text { for } E_{1}=E_{2}=0, \\
& \alpha^{2}=\sqrt{2}(4-\sqrt{13}) \text { for } E_{1}=M=0, \\
& \alpha^{2}=\sqrt{2}(4+\sqrt{13}) \text { for } E_{2}=M=0,
\end{aligned}
$$

none of which is equal to those of (69). As for Demiański's solution (68), one can see (using Maple) that its invariant $\alpha$ is never a constant (even when $b=0$ for type D; particularly for the NUT solution with $b=a=0, \alpha$ is not defined due to $\bar{r}=0$, and the corresponding CR structure is hyperquadric [7]) within the full range of the parameters $M, a$ and $b$. Therefore we conclude that the solution (69) is different from (67) and (68). 


\section{Appendix D. Kerr and Debney's type II solution}

The solution by Kerr and Debney [13] (see also [12] p. 608) admits two non-commuting Killing vectors and reads

$$
\begin{aligned}
& P_{s}=1, \quad L=A_{1} \bar{\zeta}^{2} \zeta^{1+\sigma}+A_{2} \bar{\zeta} \zeta^{\sigma / 3}, \\
& \mathrm{i} M_{s}-m_{s}=2 A_{1}(1+\sigma) \zeta^{\sigma}, \quad \Lambda=0,
\end{aligned}
$$

with $\operatorname{Re} \sigma=-3$ and $A_{1,2}$ complex constants. The special case with $\sigma=-3$ can be captured by the ansatz (42), and it corresponds to $\left(E_{1-4}\right.$ real)

$$
\begin{aligned}
& A(\zeta)=\zeta, \quad \zeta=|\zeta| \mathrm{e}^{\mathrm{i} z / 2}, \quad C_{1}=-\frac{1}{2}, \\
& F_{1}=E_{3} \sin \left(\frac{z}{2}\right)-E_{4} \cos \left(\frac{z}{2}\right)+2 E_{1} \sin \left(\frac{3 z}{2}\right)-2 E_{2} \cos \left(\frac{3 z}{2}\right), \\
& F_{2}=F_{1}^{\prime} / F_{1}, \quad F_{3}=-F_{1}^{3}\left(E_{1} \sin \left(\frac{3 z}{2}\right)-E_{2} \cos \left(\frac{3 z}{2}\right)\right), \\
& F_{4}=F_{1}^{3}\left(E_{2} \sin \left(\frac{3 z}{2}\right)+E_{1} \cos \left(\frac{3 z}{2}\right)\right), \\
& A_{1}=E_{1}+\mathrm{i} E_{2}, \quad A_{2}=E_{3}+\mathrm{i} E_{4} .
\end{aligned}
$$

Modulo some redefinition of parameters, this is the same solution as (72) and (171) with

$C_{1}=-\frac{1}{2}$. It is not clear how to make such a conversion for the general case of (D.1).

\section{References}

[1] Goldberg J N and Sachs R K 1962 A theorem on Petrov types Acta Phys. Polon. Suppl. 22 13-23

[2] Adamo T M, Newman E T and Kozameh C 2012 Null geodesic congruences, asymptotically-flat spacetimes and their physical interpretation Living Rev. Relativity 15 1-92

[3] Cartan E 1932 Sur la géométrie pseudo-conforme des hypersurfaces de l'espace deux variables complexes I Ann. Mat. 11 17-90

[4] Cartan E 1932 Sur la géométrie pseudo-conforme des hypersurfaces de l'espace deux variables complexes II Ann. Scuola Norm. Sup. Pisa $1333-54$

[5] Nurowski P 1993 Einstein Equations and Cauchy-Riemann Geometry, Ph.D. Thesis, Scuola Internazionale Superiore di Studi Avanzati, Trieste

[6] Hill C D, Lewandowski J and Nurowski P 2008 Einstein's equations and the embedding of 3dimensional CR manifolds Indiana Univ. Math. J. 57 3131-76

[7] Lewandowski J and Nurowski P 1990 Algebraically special twisting gravitational fields and CR structures Class. Quantum Grav. 7 309-328

[8] Brans C H 1965 Invariant approach to the geometry of spaces in general relativity J. Math. Phys. 6 94-102

[9] Karlhede A 1980 A review of the geometrical equivalence of metrics in general relativity Gen. Rel. Grav. 12 693-707

[10] Pollney D, Skea J E F and d'Inverno R A 2000 Classifying geometries in general relativity: I. Standard forms for symmetric spinors Class. Quantum Grav. 17 643-63

[11] Pollney D, Skea J E F and d'Inverno R A 2000 Classifying geometries in general relativity: II. Spinor tools Class. Quantum Grav. 17 2267-80

[12] Stephani H, Kramer D, MacCallum M, Hoenselaers C and Herlt E 2003 Exact Solutions of Einstein's Field Equations 2nd Ed. (Cambridge: Cambridge University Press)

[13] Kerr R P and Debney G C 1970 Einstein spaces with symmetry groups J. Math. Phys. 11 $2807-17$

[14] Zhang X and Finley D 2012 Lower order ODEs to determine new twisting type N Einstein spaces via CR geometry Class. Quantum Grav. 29065010 
[15] Nurowski P 2008 Twisting type N vacuums with cosmological constant J. Geom. Phys. 58 615-8

[16] Hanges N 1988 The missing first integral J. Diff. Eqns. 72 178-88

[17] Zhang X 2012 CR geometry and twisting type N vacuum solutions Dissertation University of New Mexico, Albuquerque

[18] Kerr R P 1963 Gravitational field of a spinning mass as an example of algebraically special metrics Phys. Rev. Lett. 11 237-8

[19] Debney G C, Kerr R P and Schild A 1969 Solutions of the Einstein and Einstein-Maxwell equations J. Math. Phys. 10 1842-54

[20] Robinson I, Robinson J R and Zund J D 1969 Degenerate gravitational fields with twisting rays J. Math. Mech. 18 881-92

[21] Krasil'shchik I S and Vinogradov A M (eds) 1999 Symmetries and Conservation Laws for Differential Equations of Mathematical Physics (Translations of Mathematical Monographs vol 182) (Providence: AMS)

[22] Nurowski P and Tafel J 1988 Symmetries of Cauchy-Riemann spaces, Lett. Math. Phys. 15 31-8

[23] Kinnersley W 1969 Type D vacuum metrics J. Math. Phys. 10 1195-203

[24] Plebański J F and Demiański M 1976 Rotating, charged and uniformly accelerating mass in general relativity Ann. Phys. 98 98-127

[25] Polyanin A D and Zaitsev V F 1995 Handbook of Exact Solutions for Ordinary Differential Equations (Boca Raton: CRC Press, Inc.)

[26] Conte R and Musette M 2008 The Painlevé Handbook (Dordrecht: Springer) (Bristol: Canopus Publishing Ltd.)

[27] Leroy J 1970 Un espace d'Einstein de type N à rayons non intégrables C. R. Acad. Sc. Paris A270 1078-80

[28] Jacobowitz H 1990 An Introduction to CR Structures (Providence: AMS)

[29] Held A 1974 A type-(3,1) solution to the vacuum Einstein equations Nuovo Cim. Lett. 11 545-6

[30] Robinson I 1975 On vacuum metrics of type (3,1) Gen. Rel. Grav. 6 423-7

[31] Lun A W-C 1978 A class of twisting type II and type III solutions admitting two Killing vectors Phys. Lett. 69A 79-81

[32] Siklos S T C 1981 Some Einstein spaces and their global properties J. Phys. A: Math. Gen. 14 395-409

[33] McIntosh C B G, Lun A W-C and Halford W D 1987 Type II and III twisting vacuum metrics and symmetries Class. Quantum Grav. 4 117-23

[34] Natorf W 2012 Asymptotic stability of vacuum twisting typ II metrics J. Math. Phys. 53022503

[35] Bičák J and Podolský J 1995 Cosmic no-hair conjecture and black-hole formation: An exact model with gravitational radiation Phy. Rev. D 52 887-95

[36] de Olivira H P and Soares I D 2004 Gravitational wave emission from the numerical evolution of Robinson-Trautman spacetimes: A treatment in the nonlinear regime Phy. Rev. D 70084041 